HIV Infection and maternal outcome of pregnancy in Mozambican Women: A case-control study

While much attention has focused on the impact of perinatal transmission of HIV infection to newborns, little attention has hitherto been given to the impact of HIV infection on maternal outcome of pregnancy. In some developing countries recent findings indicate a significantly adverse maternal outcome of pregnancy, ${ }^{1}$ whereas some studies from more affluent countries have questioned such impact. $^{2}$

In Maputo, Mozambique we have paid particular attention to genital infections during pregnancy and their impact on pregnancy outcome. We have studied five potentially infectious complications of pregnancy (threatening preterm birth, low birthweight birth, late fetal death and endometritismyometritis after vaginal, and after Caesarean delivery.

The purpose of our study was to elucidate the role of HIV-1 and HIV-2 infection in pregnant/puerperal Mozambican women suffering from any of these diseases, known to be associated with infectious complications of pregnancy.

Five different case-control studies were designed to elucidate the role of HIV-1 and HIV-2 infection in pregnant or puerperal women suffering from one of the following pregnancy complications:

Threatening preterm birth In this group 53 women with objectively confirmed painful uterine contractions and with cervix dilated less than $3 \mathrm{~cm}$ before 37 completed weeks of gestation were compared with 53 control women, without such contractions, matched for age, parity and gestational age.

Low birthweight delivery A group of 51 women with low birthweight $(<2500 \mathrm{~g})$ delivery were enrolled together with 51 control women, with normal birthweight delivery, matched for age and parity. Both cases and controls were recruited at the Maputo Central hospital.

Late fetal death In this category 58 women with confirmed intrauterine fetal death in the third trimester were recruited and compared with two groups of controls: firstly women delivering live newborns, matched for age and parity, and, secondly, undelivered women with the fetus alive, matched with the cases for age, parity and gestational length.

Endometritis-myometritis after vaginal delivery Puerperal women with (51 cases) and without (51 controls) endometritis-myometritis were enrolled, matched for age, parity and days post partum. Inclusion criteria comprised fever $\geq 30 \mathrm{C}^{\circ}$ and abnormally painful uterus.
Endometritis-myometritis after Caesarean section In the post-Caesarean section ward 47 women with endometritis-myometritis post partum were enrolled together with 47 control women, matched for age, parity and days post partum with uneventful postoperative course after Caesarean section.

Sera were taken from the cubital vein of the mother. Antibodies to HIV were analysed by a commercially available ELISA (3rd generation EIA, Abbott Laboratories, Chicago, Ill., USA). This assay detects antibodies to both HIV-1 and HIV-2. The positive reactions were confirmed by Western blot (Diagnostic Biotechnology). A total of 607 serum samples were analysed for HIV-1 and HIV-2 antibodies.

Four sera from the women studied were HIV-1 positive. No serum sample was HIV-2 positive. The positive women belonged to the following categories: (1) One case woman with threatening preterm birth. (2) One control woman in the study on low birth weight delivery. Her newborn (cord serum) was also seropositive. (3) One control woman in the study on late fetal death. (4) One control woman in the study on postCaesarean endometritis-myometritis.

In the whole group of maternal sera there were thus only $4 / 520(0 \cdot 8 \%)$ HIV-1 seropositive individuals.

Previously reported surveys among HIV-1 and HIV-2 seropositive pregnant women in Mozambique have indicated prevalences of $0.6 \%$ and $0.2 \%$, respectively. ${ }^{3}$ The present study indicates that, even in women suffering form infections leading to severe infectionassociated pregnancy pathology no increase in HIV-1 or HIV-2 seropositivity could be demonstrated.

In pregnant women with poor socioeconomic background the risk of sexually transmitted diseases and other genital infections is known to be high and such infections may adversely affect pregnancy outcome. ${ }^{4-6}$ In the present study it can be concluded that the impact of HIV seropositivity on such severe infectious morbidity among pregnant and early puerperal women in the setting studied is small or insignificant. The low prevalence of HIV infection in Mozambique may thus, at least partly, be due to the fact that vertical transmission of HIV, which is an important transmission mode in Africa, occurs only to a limited extent in the country.

STAFFAN BERGSTRÖM Department of International Health Department of International Health,
P.O. Box 1130 Blindern, N 0317 Oslo, Norway, Akademisknaecology Akademiska Hospital, ANDERS SONNNERBORG Division of Clinical Virology, 
Karolinska Institute, Huddinge Hospital, Huddinge, Sweder. NAFISSA BIQUE OSMAN AIDA LIBOMBO

Department of Obstetrics and Gynaecology, Central Hospital, Maputo,

Address correspondence to: Professor Staffan Bergström.

This study was made possible by a grant from the Swedish Agency for Research Co-operation with Developing Countries (SAREC) and the Norwegian Research Council. Both grants are gratefully acknowledged.

1 Temmerman M, Chomba EN, Ndinya-Achola J, Plummer FA, Coppens M, Piot P. Maternal human imuno deficiency virus- 1 infection and pregnancy outcome. Obstet Gynecol 1994;83:495-501.

2 Braddick MR, Kreiss JK, Embree JE. Impact of maternal HIV infection on obstetrical and neonatal outcome. HID infection on ob $1990 ; 4: 1001-5$.

3 Barreto G, Liljestrand J, Palha de Sousa C, Bergström S Böttiger G, Biberfeld G, de la Cruz F. HIV-1 and HIV-2 Böttiger G, Biberfeld G, de la Cruz F. HIV-1 and HIV-2
antibodies in pregnant women in the city of Maputo, antibodies in pregnant women in the city of
Mozambique. Scand $\mathcal{Y}$ Infect Dis 1993;23:685-8.

4 Bique-Osman N, Folgosa E, Bergström S. An inciden case-referent study of threatening preterm birth and genital infection. $\mathcal{F}$ Trop Pediatr accepted for publication 1995.

5 Bique-Osman N, Folgosa E, Bergström S. Genital infections in the etiology of late fetal death. An incident case-referent study. 7 Trop Pediatr accepted for publication 1995.

6 Libombo A, Folgosa E, Bergström S. Risk factors in puerperal endometritis-myometritis. An incident casepuerperal endometritis-myometritis. An incident case-

Accepted for publication 24 May 1995.

\section{Antiretroviral monotherapies and serum HIV-1 dynamics}

In early, frequent sampling of drug-naive patients we demonstrated characteristic responses in serum HIV-1 load with near maximal efficacy $(>90 \%)$ for zidovudine (ZDV) within 1 to 4 days of onset of treatment. ${ }^{12}$ It was self-evident to us and others that HIV-1 turnover must be extremely rapid. Making the assumptions that HIV-1 synthesis and clearance were constant and serum virus was perturbed only by antiretroviral therapy allowed us to estimate the elimination kinetics of HIV-1. ${ }^{3}$ Briefly, our "pharmacological constant infusion model" (model A) was based upon cessation (or slowing) of a drug constant infusion at steady state producing an exponential serum drug loss to zero (or to a new constant lower level) according to first order kinetics. ${ }^{4}$ By substituting virus load for drug, and onset of antiretroviral therapy for change in infusion flow, we plotted decline in serum HIV-1 against time, superimposed a model computerised curve describing this decline and estimated half-life ( $T / 2)$ by interpolation from the graphical plot (fig). This approach has been confirmed by using the same analysis upon data from drug infusion experiments (unpublished data).

Parallel, independently developed technology using an elegant computer-based mathematical model developed by Martin Nowak at Oxford described HIV-1 dynamics and its interrelationship with CD4 lymphocyte turnover..$^{5-7}$ We compared model $\mathrm{A}$ with the

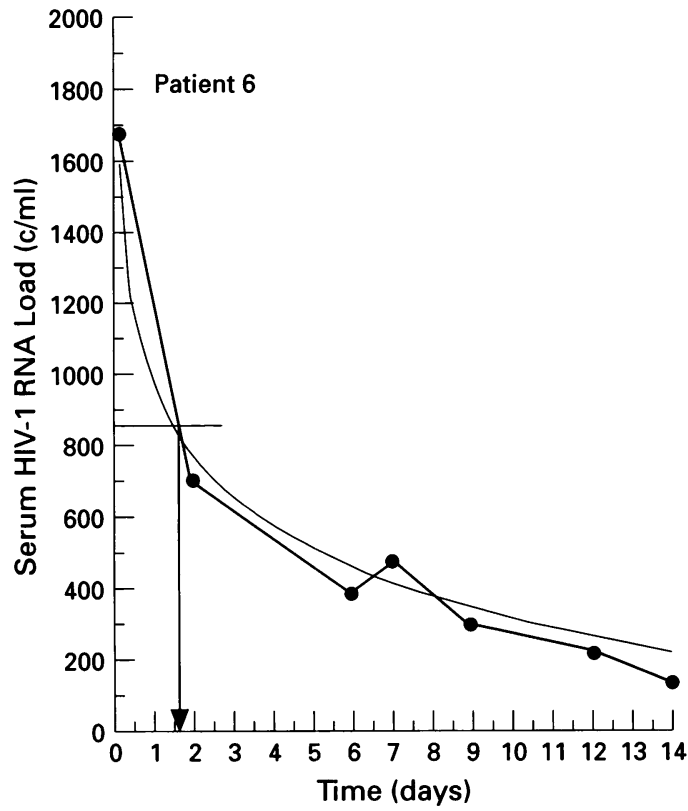

The study of serum HIV-1 dynamics using a

"pharmacological constant infusion model". In the patient shown a model computerised curve $(\longrightarrow$ ) describes the exponential decline in virus load at the onset of $Z D V$ therapy using the early serum HIV-1 RNA (copies/ml) datum points available (-), and allows the HIV-1 half-life $(T / 2)$ to be estimated $(T / 2=1.6$ days).

latter (model B) using published data from patient groups receiving single drug therapies with nucleoside reverse transcriptase (RT) inhibitors $\mathrm{ZDV},{ }^{2}$ or lamivudine (3TC), ${ }^{8}$ and protease inhibitors ABT-538 or L-735,524. ${ }^{5}$

Both models gave $T / 2$ values for $R T$ and protease inhibitors that were statistically indisguishable (table 1) and a comparison of all values derived from model $A$ and model $B$ in 39 patients produced a linear relationship with a regression coefficient of 0.77 ( $p<$ $0.001)$. Using either approach no monotherapy examined appeared to be superior in terms of early efficacy estimated by $T / 2$ values.

Either of these models may be used to study serum/plasma HIV-1 dynamics. However, two important caveats must be emphasised in relation to such analyses.

$A$ comparison of the two models (in-house: Model $A$ and Martin Nowak: Model B) for estimating serum/plasma $H I V-1$ half-life (T/2) in days at the onset of antiretroviral monotherapies

\begin{tabular}{llll}
\hline & $\begin{array}{l}\text { Model } A \\
\text { Mean time } \\
(+/-s d) \\
\text { days }\end{array}$ & $\begin{array}{l}\text { Model } B \\
\text { Mean time } \\
(+/-s d) \\
\text { days }\end{array}$ & $\begin{array}{l}\text { Stat. } \\
\text { sig. }\end{array}$ \\
\hline $\begin{array}{l}\text { ZDV } \\
(\mathrm{n}=10)\end{array}$ & $1 \cdot 7(0 \cdot 7)$ & $1.9(1 \cdot 0)$ & NS \\
$\begin{array}{l}\text { 3TC } \\
(\mathrm{n}=18)\end{array}$ & $1 \cdot 6(1 \cdot 1)$ & $2 \cdot 1(1 \cdot 5)$ & NS \\
$\begin{array}{l}\text { ABT-538 } \\
(\mathrm{n}=7)\end{array}$ & $2 \cdot 0(0 \cdot 4)$ & $2 \cdot 4(0 \cdot 5)$ & NS \\
$\begin{array}{l}\text { L-735,524 } \\
(\mathrm{n}=4)\end{array}$ & $2 \cdot 1(0.7)$ & $2 \cdot 4(0 \cdot 6)$ & NS \\
\hline
\end{tabular}

sd $=$ standard deviation.

$\mathrm{n}=$ patient number per group.

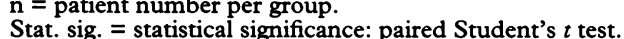
NS = Not significant. 